\title{
Empirical relations for cluster RR Lyrae stars revisited
}

\author{
G. Kovács ${ }^{1}$ and A. R. Walker ${ }^{2}$ \\ 1 Konkoly Observatory, PO Box 67, 1525 Budapest, Hungary \\ 2 Cerro Tololo Inter-American Observatory, National Optical Astronomy Observatory^, Casilla 603, Chile \\ e-mail: awalker@noao.edu
}

A\&A, 371, 579-591 (2001), DOI: 10.1051/0004-6361:20010346

Because of an unfortunate mistake, a rough reddening correction from earlier works was left in the average $V$ magnitudes of the variables of M 4 . Therefore, the reddened distance modulus $\left(\left\langle\tilde{d}_{V}\right\rangle\right)$ for this cluster is incorrectly given in Table 9 . The correct value of $\left\langle\tilde{d}_{V}\right\rangle$ is -4.43 instead of -5.69 as given in the paper. The above mistake does not affect any other parts of the paper, including the dereddened distance moduli of M 4.

\footnotetext{
Send offprint requests to: G. Kovács, e-mail: kovacs@konkoly.hu

* The National Optical Astronomy Observatory is operated by the Association of Universities for Research in Astronomy, Inc., under cooperative agreement with the National Science Fundation.
} 